\title{
To be, or not 3B
}

\author{
Joo Y. Song \\ Department of Pathology, City of Hope National Medical Center, Duarte, CA, USA \\ E-mail: josong@coh.org \\ doi:10.3324/haematol.2021.280263
}

Although follicular lymphoma $(\mathrm{FL})$ is the most common, indolent non-Hodgkin B-cell lymphoma, grade 3B (FL3B) only accounts for $5-10 \%$ of cases of $F L$ and is characterized by follicles consisting exclusively of centroblasts. ${ }^{1} \mathrm{Ap}-$ proximately half of the cases of FL3B also show a low-grade component (grade 1-2) or diffuse large B-cell lymphoma (DLBCL). ${ }^{2}$ Most consider FL3B to be aggressive and treat these cases as DLBCL with intense immunochemotherapy such as rituximab plus cyclophosphamide, doxorubicin, vincristine and prednisone (R-CHOP). However, there are limited data on these cases from molecular and therapeutic standpoints since these cases are often excluded from clinical trials.

In this issue of Haematologica, Koch and colleagues ${ }^{3}$ help to shed light on this rare and aggressive disease by analyzing cases of FL3B alone and FL3B together with DLBCL using a targeted sequencing panel and taking care to exclude cases of pediatric FL and cases with the IRF4 translocation. ${ }^{4}$ The authors observed that $88 \%(14 / 16)$ of the cases of composite FL3B and DLBCL showed mutational variants shared by the FL3B component and DLBCL after macrodissection of these areas. In addition, the majority of the cases shared the same chromosomal abnormalities with BCL2, BCL6, and MYC rearrangements by fluorescence in situ hybridization analysis. These findings are similar to those of other studies evaluating the genomic landscape of FL and its transformed state,${ }^{5}$ as there were no specific recurrent mutations identified to predict transformation to DLBCL. Not surprisingly, the most frequent mutations that were shared by the FL3B and DLBCL components were CREBBP and KMT2D (Figure 1).

The next question is how do patients with FL3B respond to intense chemotherapy? In a subset of the patients with FL3B and FL3B plus DLBCL who were enrolled in the prospective randomized PETAL trial, ${ }^{6}$ analysis of overall and progression-free survival showed that, in this limited cohort of patients, the survival outcomes of these patients were similar to those of patients with de novo DLBCL.

FL3B continues to be a difficult disease to analyze from genomic and clinical standpoints. The difficulties are due in part to the absence of these cases in large, prospective clinical trials, the heterogeneity of composite disease with low-grade FL or DLBCL, and issues with poor reproducibility of grading. Koch and colleagues ${ }^{3}$ show that the genomic abnormalities in cases of concurrent FL3B and $D L B C L$ are shared by the two components, further supporting the concept that FL3B may represent DLBCL and can be managed with aggressive immunochemotherapy in a manner similar to de novo DLBCL. The retained follicular

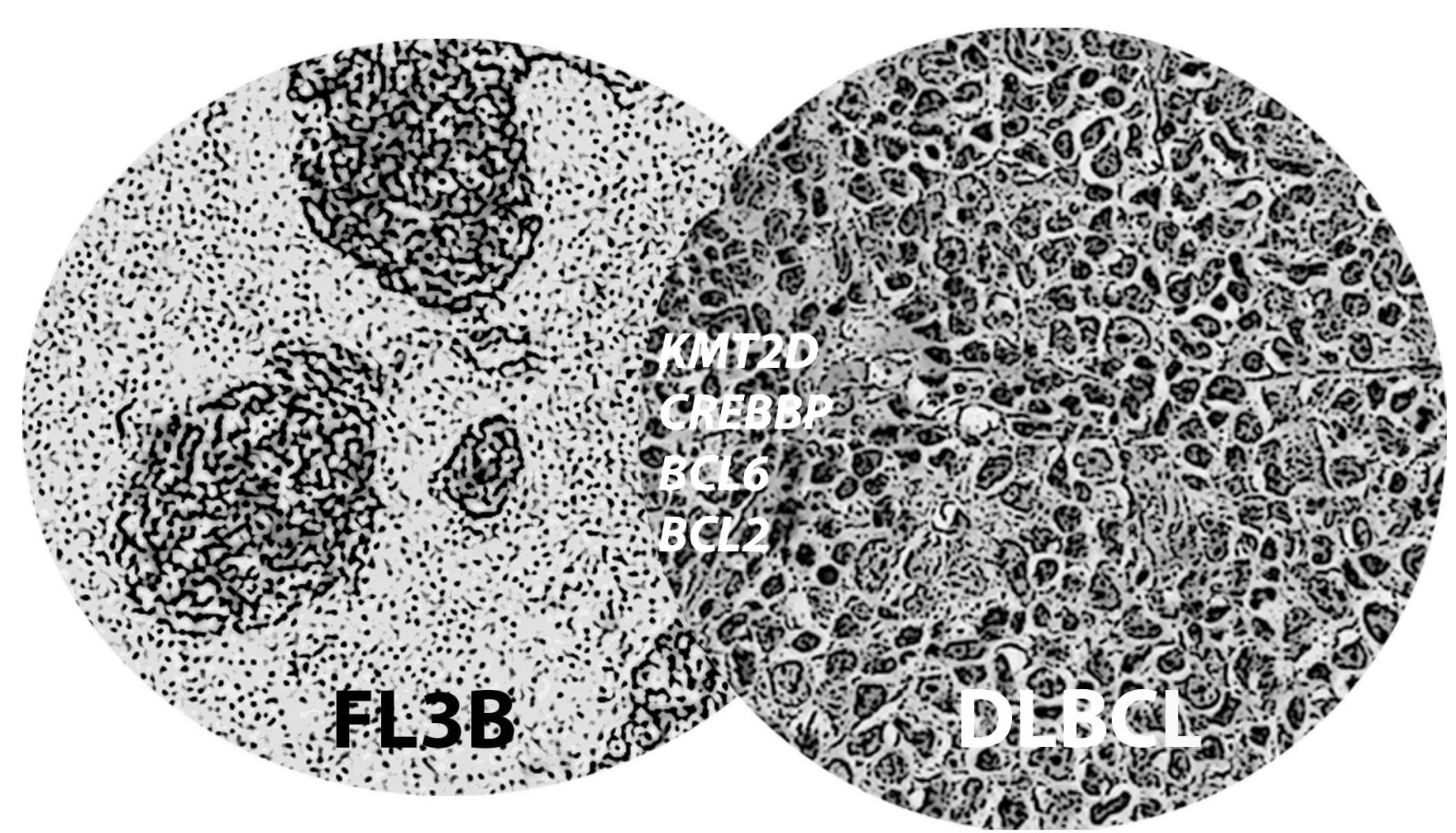

Figure 1. Follicular lymphoma grade 3B and diffuse large B-cell lymphoma. Left: follicular lymphoma grade 3B (FL3B) with an expanded follicular dendritic cell meshwork highlighted with CD21. Right: diffuse large B-cell lymphoma (DLBCL) with sheets of large and atypical cells. Concurrent FL3B and DLBCL show shared mutations and translocations with KMT2D, CREBBP, BCL6, and BCL2. 
structure in FL3B may be related to the tumor microenvironment or an early snapshot of $\mathrm{FL}$ progressing to DLBCL. It is unclear whether cases of FL with development of DLBCL at a later time-point would also have many shared mutations like these composite cases. Clinical trials have recently started to include FL3B, as seen with the TRANSCEND NHL 001 trial, investigating antiCD19 chimeric antigen receptor $\mathrm{T}$ cells. Another study, using selinexor plus chemotherapy for refractory aggressive B-cell lymphomas (NCT02471911), provides some promise for inclusion of this rare disease in future studies. The study by Koch and colleagues ${ }^{3}$ further underscores that FL3B may be a continuum of DLBCL with current evidence pointing towards grouping as an aggressive lym- phoma. Cases with only FL3B may be a result of limited sampling with DLBCL being present in less surgically accessible sites. Mutational profiling has yet to provide a mechanism of disease progression and more cases of paired $F L$ and DLBCL are required to address these issues. Although there are challenges in studying FL3B due to the rarity of the disease, inclusion of FL3B in prospective trials, with steps to study the combination with genomic correlatives, will help to clarify how to define the disease better. This, in turn, will help to aid the process towards selecting appropriate treatment.

\section{Disclosures}

No conflicts of interest to disclose.

\section{References}

1. Barraclough A, Bishton M, Cheah CY, Villa D, Hawkes EA. The diagnostic and therapeutic challenges of grade 3B follicular lymphoma. Br J Haematol. 2021;195(1):15-24.

2. Ott G, Katzenberger T, Lohr A, et al. Cytomorphologic, immunohistochemical, and cytogenetic profiles of follicular lymphoma: 2 types of follicular lymphoma grade 3. Blood. 2002;99(10):3806-3812.

3. Koch C, Richter J, Hänel C, Hüttmann A, Dührsen U, Klapper W. Follicular lymphoma grade 3B and diffuse large B-cell lymphoma present a histopathological and molecular continuum lacking features of progression/transformation. Haematologica. 2022;107(9):2144-2153.
4. Swerdlow S, Campo E, Harris NL, et al. WHO Classification of Tumours of Haematopoietic and Lymphoid Tissues. Lyon, France: International Agency for Research on Cancer, 2017.

5. Bouska A, Zhang W, Gong Q, et al. Combined copy number and mutation analysis identifies oncogenic pathways associated with transformation of follicular lymphoma. Leukemia. 2017;31(1):83-91.

6. Dührsen U, Müller S, Hertenstein B, et al. Positron emission tomography-guided therapy of aggressive non-Hodgkin lymphomas (PETAL): a multicenter, randomized phase III trial. J Clin Oncol. 2018;36(20):2024-2034. 